\title{
Dificultades sociales y educativas de los niños y niñas con cáncer y sus familias. Una visión desde el trabajo social en Oncología Pediátrica de Galicia Social and educational problems of children with cancer and their families. A
view from Galician Pediatric Oncology Social Workers.
}

\author{
Francisco Xabier Aguiar Fernández, Andrea Pazos Baña \\ Universidade de Vigo
}

\begin{abstract}
Resumen
La hospitalización es una de las principales fuentes de estrés de los niños y niñas diagnosticados de cáncer debido a la ruptura forzada con el ambiente familiar y escolar. En esta comunicación nos proponemos identificar los factores sociales presentes y su interrelación con el proceso educativo. Se ha realizado una investigación de tipo cualitativo mediante la técnica de la entrevista a trabajadoras sociales de los Servicios de Oncología Pediátrica de Galicia y a miembros del movimiento asociativo. Los resultados muestran una escasez de recursos de atención social y de apoyo al seguimiento escolar y educativo.

Palabras clave: cáncer, pediatría, trabajo social, educación, derechos
\end{abstract}

\begin{abstract}
Hospitalization is one of the main sources of stress for children diagnosed with cancer due to forced rupture with the family and school environment. In this paper we intend to identify the social factors present and their interrelation with the educational process. A qualitative research has been carried out using the technique of interviewing social workers of the Pediatric Oncology Services of Galicia and members of the associative movement. The results show a shortage of resources for social care and support for school and educational monitoring.

Keywords: Cancer, pediatrics, social work, education, rights
\end{abstract}

\section{Introducción}

El cáncer y sus tratamientos suponen un acontecimiento traumático y estresante para los/las niños/as y sus familias. Durante el curso de la enfermedad se exponen a situaciones potencialmente estresantes como la amenaza para la vida o la integridad física, procedimientos médicos invasivos y dolorosos, importantes efectos secundarios derivados de los tratamientos, cambios físicos y funcionales, frecuentes hospitalizaciones, alteración de la rutina cotidiana y de la dinámica familiar, social y escolar (Bragado, 2009; Palomo, 1999). Asimismo, el/la niño/a se enfrenta a diversas emociones como la soledad, el miedo, la ira, la ansiedad y la depresión. El nivel de desarrollo del niño/a determina la naturaleza del impacto emocional y las habilidades que usará para afrontar el cáncer (Baquero y Ibáñez, 2009; Espada, López-Roig, Méndez y Orgilés, 2004). Por lo tanto, su vida y la dinámica familiar se transforman bruscamente, iniciándose un nuevo período de adaptación a personas, situaciones y ambientes desconocidos. Sin embargo, no hay que olvidar que el cáncer es un hecho circunstancial en la vida del niño/a y que éste continúa con su proceso madurativo y evolutivo, por lo que resulta relevante la integración de la enfermedad en su vida normal y en la de su familia (López-Ibor, 2009).

La familia influencia en gran medida el impacto y el ajuste de la infancia al cáncer (Chaskel y Salcedo, 2012). En concreto, el diagnóstico de esta enfermedad está considerado como uno de los acontecimientos más estresantes para el entorno familiar y algunos/as autores/as lo definen como una enfermedad familiar (De la Garza, 2007).Las madres y los padres no responden de la misma forma ante la enfermedad del niño/a. Las reacciones de cada familia ante el diagnóstico están influidas por el sistema de creencias familiares, la personalidad de sus miembros, la experiencia familiar en enfermedades, el momento evolutivo que atraviesa la familia, los propios factores de la enfermedad (comienzo y curso, entre otros) y los sistemas de apoyo con los que cuenta (Grau, 2004; Herrero, 2006). Las familias dejan de tener tiempo para estar con las amistades y para el ocio, las relaciones se empobrecen, se deteriora la comunicación y disminuyen los apoyos personales y sociales perjudicando el proceso de adaptación familiar a los cambios (Lanzarote y Torrado, 2009). El aislamiento puede intensificarse si la familia percibe que sus amistades y familiares no saben cómo reaccionar y evitan hablar del tema (Grau, 2004; Herrero, 2006).

En referencia a los problemas sociales en la comunidad, podemos afirmar que el principal es el absentismo escolar (Grau y Ortiz, 2001). Para Guijarro (2010), el proceso de socialización es determinante en los diferentes estadios o fases del desarrollo infantil. Por 
este motivo, resulta de gran relevancia el apoyo al niño/a, no sólo por parte de sus familiares y del personal sanitario, sino también por parte de la escuela (Espada et al. 2004). La enfermedad produce inestabilidad en las relaciones sociales con amistades y camaradas, debido a los largos periodos de absentismo escolar, y aumenta el riesgo de sufrir problemas en sus relaciones sociales y en su autoestima debido a las alteraciones físicas (Grau, 2002).

A pesar de las dificultades que impiden la asistencia al colegio, continuar con sus actividades sociales y académicas les ofrece la oportunidad de normalizar una experiencia tan estresante como es la enfermedad debido a que "la percepción de apoyo social por parte de los/las compañeros/as es un predictor consistente en el proceso de adaptación" (Colegrove, Dolgin, Katz y Varni, 1994, como se cita en Guijarro, 2010, p. 78), y cuando perciben bajo apoyo social se aumenta el riesgo de que surjan dificultades de adaptación (Espada et al., 2004). Por esta razón, durante la hospitalización es necesaria la asistencia a las aulas hospitalarias, siempre que su estado de salud lo permita, ya que permiten la normalización de la vida. Del mismo modo, posibilitan la continuidad del proceso de aprendizaje; compensando así el posible retraso académico, la ocupación y distracción durante los tiempos que tienen libres, la promoción de la confianza e independencia en el medio hospitalario, favoreciendo la integración socioafectiva con otros/as niños/as; evitando que se produzca el aislamiento, y la prestación de un apoyo emocional (Grau, 2004; Grau y Ortiz, 2001).

Hoy en día, los tratamientos médicos suelen realizarse en régimen ambulatorio o de asistencia domiciliaria, para reducir así los períodos de hospitalización. Por esta razón, los/las niños/as en algunos casos suelen permanecer en el domicilio sin poder acudir a la escuela, por lo que se hace necesaria la asistencia de un/a maestro/a al domicilio para continuar con el proceso educativo, en coordinación con el profesorado del centro educativo y con el aula hospitalaria (Grau, 2004).

La mayoría de los casos cuando ingresan en un hospital están escolarizados, por lo que al ingresar rompen con esta actividad escolar que les es propia. La escuela en el hospital trata de mitigar los efectos de esta ruptura. Siguiendo a Lizasoain y Ochoa (2003), el modo de actuación a través de la pedagogía hospitalaria lo podemos dividir en cuatro grandes grupos: enseñanza escolar, actividades lúdico-recreativas, orientación personal y familiar y estrategias psicopedagógicas específicas de intervención.

En el año 1986 el Parlamento Europeo aprobó la resolución sobre la Carta Europea de los Niños Hospitalizados y en 1988 tuvo lugar en Eslovenia el primer seminario europeo sobre "la educación del niño hospitalizado", bajo el patrocinio de la UNESCO y la OMS. En el año 2000, en el marco de un congreso celebrado en Barcelona, la HOPE (Hospital Organisation of Pedagogues in Europe), asociación internacional con fines científicos y educativos, presentó la Carta Europea sobre el Derecho a la
Atención Educativa de los Niños y Adolescentes Enfermos.

En la Comunidad Autónoma de Galicia, el Decreto 229/2011, de 7 de diciembre, por el que se regula la atención a la diversidad del alumnado de los centros docentes en los que se imparten las enseñanzas establecidas en la Ley orgánica 2/2006, de 3 de mayo, de educación, establece en su artículo 25 que la atención educativa hospitalaria y/o domiciliaria tendrán por finalidad la continuidad del proceso formativo del alumnado que curse enseñanzas de régimen general en modalidad presencial en centros sostenidos con fondos públicos y que, por prescripción facultativa no pueda asistir con regularidad al centro por un período prolongado de tiempo. Será destinatario de atención educativa hospitalaria el alumnado que deba permanecer ingresado en un centro hospitalario, a tiempo completo o en hospitalización de día, en alguna de las siguientes situaciones: larga hospitalización (más de treinta días), media hospitalización (entre quince y treinta días) y corta hospitalización (menos de quince días). Así mismo, será destinatario de atención educativa domiciliaria el alumnado que deba permanecer convaleciente en el domicilio por un período de tiempo superior a un mes o que padezca alguna enfermedad crónica que sea la causa de faltas de asistencia al centro de seis o más días continuados al mes dentro de un período mínimo de seis meses.

El trabajo social sanitario forma parte del equipo de atención psicosocial presente en el Servicio de Oncología Pediátrica e investiga los factores psicosociales que influyen en el proceso de salud-enfermedad (Colom, 2008; Ituarte, 2012). Esto permite tanto al equipo sanitario como al profesional del trabajo social la profundización y el conocimiento global de cada paciente, adaptándose a la realidad y a las características particulares de la enfermedad para poder desarrollar la intervención social (Novellas, 2004; Rodríguez, 2014).

En esta comunicación nos proponemos abordar el impacto de la enfermedad en el contexto familiar y comunitario e identificar los factores sociales presentes, con especial atención a su repercusión e interrelación con el proceso educativo.

\section{Método}

El presente estudio de investigación cualitativa de tipo exploratorio-descriptivo se ha realizado mediante la técnica de la entrevista semiestructurada. Tomando como referencia la Comunidad Autónoma de Galicia, se han entrevistado a 5 profesionales del Trabajo Social de los cinco hospitales gallegos del Servicio Gallego de Salud (SERGAS) donde existen Unidades o Servicios de Oncología pediátrica: Hospital Clínico de Santiago de Compostela; Hospital materno-infantil Teresa Herrera (A Coruña); Hospital Alvaro Cunqueiro (Vigo); Hospital Santa María Nai (Ourense) y Hospital Lucus Augusti (Lugo). También se ha entrevistado a una trabajadora social de atención primaria de salud con amplia experiencia en pediatría y a otra de un centro estatal de referencia en Oncología pediátrica (Valencia). 
La muestra se ha completado con una entrevista a una profesional de la Asociación Española contra el Cáncer en Galicia (AECC) y a un miembro/a de la Asociación de Ayuda a Niños Oncológicos de Galicia (ASANOG).

Los datos fueron transcritos bajo un diseño narrativo y se categorizó el contenido (Martínez, 2006), a partir de los bloques temáticos establecidos en la investigación, para posteriormente proceder a su análisis e interpretación.

\section{Resultados}

En referencia a la percepción de los procesos de hospitalización y los tratamientos médicos a los que la infancia con cáncer es sometida durante el curso de la enfermedad, los/las profesionales expresan que ésta se ve inmersa repentinamente en un medio cerrado, siendo habituales las reacciones de rabia, de rechazo, de incompresión y de miedo, ligadas al hecho de que en un principio en esta etapa vital no se han tenido demasiadas experiencias asociadas con el dolor y el malestar. Entre las situaciones que se señalan más difíciles de sobrellevar nos encontramos con los procesos de hospitalización que conllevan el aislamiento por medidas de precaución y algunos tratamientos de forma ambulatoria que implican efectos secundarios agresivos y dolorosos.

Las actividades sociales y escolares en el hospital se presentan como un factor de gran importancia que favorece la integración. Todos/as los/las profesionales subrayan que es algo fundamental, puesto que es el modo que tienen de normalizar su vida y romper con la rutina hospitalaria, mediante la integración de aspectos relacionados con el juego, con actividades educativas y con la apertura al exterior de visitas durante el proceso de enfermedad, para que no se produzca una desvinculación con el exterior y con su vida.

"[...] es básico que los niños mantengan y tengan aquí sus espacios lúdicos, normalizar su vida es que tengan y mantengan sus contactos $y$ sus programas escolares"E.2.

En la mayoría de los casos son las madres las que asumen el rol de cuidadora primaria. No obstante, señalan que generalmente cuando en el núcleo familiar existen ambos progenitores, la presencia de los padres y su implicación es importante. Los casos en que el cuidador principal es el padre siguen siendo un número reducido. Sobre todo al comienzo del proceso, son muchas las parejas que intentan solicitar bajas laborales para poder estar presentes en el hospital, aunque esto no es siempre posible ya que es una cuestión sujeta a la permisividad laboral. Cuando la enfermedad implica a la infancia, el despliegue familiar, tanto de la familia nuclear como extensa, presenta matices propios, y parece existir mayor apoyo al cuidador principal y una rotación de cuidadores.

En lo que respecta, a las principales dificultades familiares surgidas durante la enfermedad a raíz de los procesos de hospitalización y las visitas hospitalarias, los/las profesionales coinciden que giran en torno a los siguientes puntos:
-La “cuestión sanitaria”: las preocupaciones se focalizan en la situación de salud, es decir, la incertidumbre por el diagnóstico, la información recibida, la ansiedad producida por el retraso en una prueba o los viajes a otros hospitales.

-Las relaciones sociales: se suspenden o incluso se eliminan, siendo habitual que se produzca el aislamiento social en estas familias.

-Reorganización del proyecto de vida: implica cuestiones como la paralización en la actividad laboral, saber quién va a tener disponibilidad de su tiempo para cuidar.

- Conciliar cuidado de otros/as hijos/a con el cuidado del niño/a que está en el hospital: a veces, incluso aparece el sentimiento de culpa, porque sienten que durante la atención al niño/a dejan de lado a los/las hermanos/as.

-La descoordinación entre centros y servicios en los tratamientos: consideran que es inadmisible que cuando son derivados a otros hospitales, se vean en la obligación de deambular para la realización de pruebas, analíticas y resonancias, así como para acudir a consultas de determinados servicios. En este sentido es consensual la idea de que la atención se debería prestar desde una unidad coordinada con todos los dispositivos necesarios y desde donde se preste una atención integral.

"[...] os pais convirtense en auténticos profesionais das ventás de citación, e iso é o que non pode ser. Ten que haber unha única unidade que centralice todas estas cuestións [...] que se lle dea unha atención global e non só dos aspectos médicos, senon que tamén se aborden os aspectos psicolóxicos e socias" E.4.

En las entrevistas se señaló que uno de los problemas mayoritarios a los que se enfrentan estas familias son los económicos y laborales. El primero de ellos, hace referencia a las nuevas necesidades económicas surgidas a raíz de la enfermedad, y comprende los gastos extraordinarios por tener que estar durante largos períodos de tiempo en el hospital, las dietas, los desplazamientos o el alojamiento. En ocasiones es alguna Fundación quien se hace cargo de esto último, pero siempre existen costes añadidos como es el caso de algunas medicinas no financiadas. También supone un gasto el tener que contratar a una persona para el cuidado de otros/as hijos/as o de otras personas dependientes.

"[...] en familias que son más vulnerables o que están un poco más al límite hay mayor dificultad”. E.2

Otra de las grandes dificultades señaladas deriva de las limitadas políticas y recursos de apoyo a la conciliación. Existe una prestación por cuidado de menores afectados por cáncer u otra enfermedad grave que permite el cobro del $100 \%$ del salario por reducción de jornada, pero uno de los criterios que sigue dicha prestación es que ambos progenitores estén trabajando, y sólo puede ser uno de los dos quien acceda a la prestación o bien se alternen. Aquellas familias con progenitores en desempleo sólo contarían con un sueldo o no pueden acceder a la prestación. "[...] todo está relacionado con la situación laboral y que tipos de contratos tenemos. En la mayoría de las ocasiones 
supone elementos negativos añadidos a la situación de enfermedad [...]". E.6

En las entrevistas se consideró de especial relevancia la presencia de las aulas hospitalarias en todos aquellos hospitales donde haya un servicio de Pediatría para la realización, no solo de actividades formativas, sino también lúdicas y de animación. El objetivo es aminorar los efectos de la ruptura en la vida social y conservar el contacto con los compañeros/as, sobre todo en la adolescencia. "[...]la ruptura con el medio escolar, el absentismo justificado es un problema, sobre todo para los mayores. Evidentemente ante un tema de salud no es lo prioritario, pero si lo es para su normalización y para sentirse que llevan su vida como sus iguales [...]" E.2.

Los/as profesionales han detectado problemas de acceso y cobertura en el sistema de escolarización a domicilio. De hecho, denuncian la falta de un sistema adecuado por parte de la Consellería de Educación en Galicia que permita compensar el absentismo escolar. La presión de una madre que denunció en los medios de comunicación esta situación suscitó mayor interés y medidas al respecto por parte de la administración gallega. " [...] isto é o que non pode ser, as nais teñen bastante máis de que preocuparse que de ter que saír na prensa continuamente para esixir que o seu fillo poida continuar cunha escolarización a domicilio." E.4.

En lo que respecta a la existencia de algún protocolo y/o programa de intervención con el/la niño/a con cáncer y sus familias, se indicó que actualmente no existen en ninguno de las Unidades y Servicios de Trabajo Social, con lo cual se ve resentida la coordinación con otros servicios del hospital o de la comunidad, como es el caso de los centros educativos. En alguno de los hospitales se ha detectado que en el momento del estudio se encontraba en fase de elaboración. También mayoritariamente las y los profesionales manifestaron la carencia de protocolos de intervención propio del equipo que conforma la Unidad de Oncología Pediátrica. Sin embargo, existen protocolos médicos y de enfermería.

Las dificultades que las personas entrevistadas se encuentran durante el proceso de intervención son diversas y numerosas, pero coinciden en señalar el propio estado emocional de las familias debido al impacto que supone el diagnóstico. Además, la integración en el Servicio llega a ser un problema, ya que no pueden acceder a todas las familias y además disponen de poco tiempo para dedicarles. Las políticas de austeridad y los recortes en sanidad de los últimos años han impedido la incorporación de un mayor número de profesionales del trabajo social a las instituciones sanitarias del SERGAS. Se ha detectado una sobrecarga de trabajo que impide una atención de mayor calidad, el poder trabajar a través de protocolos preventivos o el coordinarse en mayor medida con otro tipo de dispositivos y profesionales, entre ellos los del ámbito educativo. Además denuncian que las ayudas y los recursos económicos, humanos y materiales destinadas a favorecer el seguimiento escolar y la participación en la comunidad son insuficientes.

\section{Discusión}

El diagnóstico del cáncer supone un gran impacto emocional, no sólo para el/la niño/a, sino para todo su entorno familiar (De la Garza, 2007; Lizasoáin y Ochoa, 2003 y Guijarro, 2010). Coincidiendo con la clasificación elaborada por Giammona y Malek (2002, citado en Torrado, 2015), los/las profesionales señalan que el impacto en el/la niño/a está determinado por dos factores como son la edad y la madurez.

En relación al absentismo escolar muchos profesionales mantienen que es un problema social en la línea de lo manifestado por autores como Grau y Ortiz (2001) y Espada et al. (2004). En nuestro estudio se manifestó que la integración de las actividades sociales y escolares en el hospital es algo fundamental puesto que coincidiendo con Grau (2004) es una forma de poder normalizar su vida y continuar con el proceso de aprendizaje.

Los principales problemas señalados en relación a la hospitalización y a la enfermedad en general son económicos, laborales, de organización familiar, de relaciones sociales, de conciliación del cuidado y emocionales, tal y como afirma Muniáin (2003). Las funciones realizadas por los/las profesionales del trabajo social con el equipo sanitario son la información y aportación de datos sobre las situaciones socio familiares que influyen en el proceso de salud-enfermedad (Ituarte,2012; Novellas, 2004). En nuestro estudio hemos visto que actualmente no existe ningún protocolo o programa de intervención del trabajo social con el/la niño/a con cáncer y sus familias en los hospitales gallegos. Sin embargo, se consideró de importancia su creación e implantación en la línea de lo sugerido por Muniáin (2003), con el objetivo de aunar los criterios de intervención de los/las trabajadores sociales de la Federación Española de Padres de Niños con Cáncer. También se señaló la necesidad de una mayor formación específica (Gil y Novellas , 2004), y de una intervención que tenga más en cuenta el interés de la infancia y la coordinación con otros dispositivos y centros educativos de la comunidad.

\section{Conclusiones}

El Trabajo Social en Oncología no debe focalizarse sólo en los elementos de apoyo y acompañamiento durante el proceso, sino en movilizar redes sociales de cara a conseguir la transformación de la realidad social. En este sentido, consideramos que la actuación debe apostar por un enfoque de derechos y por una visión enfocada en la infancia. Con ello nos referimos a que las intervenciones deben estar focalizadas en el desarrollo de las capacidades de las personas para que puedan hacer efectivos sus derechos. Esta es la manera de conseguir una disminución de las desigualdades y la forma de posibilitar el disfrute de la vida plena de las personas. En este trabajo pudimos comprobar como los/las profesionales debido a la falta de integración en el equipo, la sobrecarga de trabajo derivada de la insuficiencia de recursos humanos y la falta de prestaciones disponibles para la infancia con cáncer y sus familias, se ven con dificultades para abarcar en 
mayor medida funciones de coordinación o actividades que se deberían llevar a cabo en la práctica, y que limitan las posibilidades de intervenir en aquellos factores sociales que dificultan el ejercicio del derecho a la educación o a participar en la comunidad.

\section{Referencias}

Baquero, A., \& Ibánez, E. (2009). Beneficio del apoyo psicosocial a la calidad de vida de niños y niñas enfermos de cáncer: una revisión sistemática cualitativa. Revista colombiana de enfermería. Recuperado de http://www.uelbosque.edu.co/publicaciones/revista_c olombiana_enfermeria/volumen-iv-numero-4

Bragado, C. (2009). Funcionamiento psicosocial e intervenciones psicológicas en niños con cáncer. Psicooncología, 6, 327-341.

Chaskel, R., \& Salcedo, C.M. (2012). Impacto psicosocial del cáncer en niños y adolescentes. CCAP, 8 (3), 5-18. Recuperado de https://scp.com.co/precop-old/precop_files/modulo_8 _vin_3/Impacto_Psicosocial_del_Cancer.pdf

Colom, D. (2008). El trabajo social sanitario. Atención primaria y atención especializada. Teoría y práctica. Madrid, España: Siglo XXI.

De la Garza, F. (2007). Cáncer: Guía para pacientes y familiares. Sevilla, España: Trillas.

Espada, J.P., López-Roig, S., Méndez, X., \& Orgilés, M. (2004). Atención psicológica en el cáncer infantil. Psicooncología, 1 (1), 139-154. Recuperado de https://dialnet.unirioja.es/servlet/articulo? codigo $=170$ 5535

Gil, F., \& Novellas, N. (coords.) (2004). Modelo de atención psico-social en oncología: standards. Psicooncología, 1 (1), 179-184.

Grau, C. (2002). Impacto psicosocial del cáncer infantil en la familia. Educación, Desarrollo y Diversiad, 5 (2), 87-106. Recuperado de: http://mobiroderic.uv.es/bitstream/handle/10550/4163 9/004320.pdf?sequence $=1$ \&isAllowed $=y$

Grau, C. (2004). Atención educativa al alumnado con enfermedades crónicas o de larga duración. Málaga, España: Aljibe.

Grau, C., \& Ortiz, M.C. (2001). Las necesidades educativas especiales derivadas de enfermedades crónicas y de larga duración. Enciclopedia psicopedagógica de necesidades educativas especiales, 2, 169-186. Recuperado de https:/www.researchgate.net/profile/Claudia_Rubio/p ublication/272706790_Atencin_educativa_a_las_nece sidades_educativas_especiales_derivadas_de_enferme dades_crnicas_y_de_larga_duracin/links/54ec65240cf $2465 \mathrm{f} 532 \mathrm{e} 88 \mathrm{c} 9 . \mathrm{pdf}$

Guijarro, A. (2010).Estructura y funcionamiento familiar de niños con cáncer desde la perspectiva de las madres. (Tesis doctoral). Universidad de la Laguna, Tenerife.

Herrero, M. (2006). El sistema familiar afectado por la enfermedad y la deficiencia. En M. Fernández, y I. García (Eds.), Orientación familiar. Familias afectadas por enfermedad o discapacidad (pp.30-33). Burgos, España: Universidad de Brugos.

Ituarte, A. (2012). Cuestiones básicas en el proceso clínico de atención psicosocial. Trabajo Social y Salud, 72, 5-36.

Lizasoáin, O., \& Ochoa, B. (2003). Repercusiones familiares originadas por la enfermedad y la hospitalización pediátrica. Osasunaz, (5), 87-99.

Lanzarote, M.D., \& Torrado, E. (2009). Orientación y psicoterapia familiar con niños y adolescentes con enfermedad crónica. Apuntes de psicología, 27 (2-3), 457-471. Recuperado

de http://www.cop.es/delegaci/andocci/files/contenidos/v ol.\%2027\%20n\%BA\%202-3,\%202009\%20apuntes\% $20 \mathrm{psicolog} \% \mathrm{EDa} / 20 . \mathrm{pdf}$

López-Ibor, B. (2009). Aspectos médicos, psicológicos y sociales del cáncer infantil. Psicooncología, 6 (2-3), 281-284. Recuperado de https://dialnet.unirioja.es/servlet/articulo? $\operatorname{codigo}=311$ 3769

Muniáin, A.R. (2003). El niño con cáncer. Problemática social. Osasunaz, (5), 101-120. Recuperado de http://www.euskomedia.org/PDFAnlt/osasunaz/05/05 101120.pdf

Martínez, M. (2006). La investigación cualitativa (Síntesis conceptual). Revista de investigación en psicología, 9 (1), 123-146.

Novellas, A. (dir.). (2004). Modelo de trabajo social en la atención oncológica. Recuperado de http://ico.gencat.cat/web/.content/minisite/ico/professi onals/documents/qualy/arxius/doc_modelo_trabajo_so cial_at._oncologica.pdf

Palomo, M. P. (1999). El niño hospitalizado. Madrid, España: Pirámide.

Rodríguez, A.M. (2014). Papel del trabajador/a social con enfermos/as oncológicos/as sometidos/as a tratamiento en una unidad de radioterapia. Trabajo Social y Salud, 77, 75-80.

Torrado, E. (2015). Familias con un hijo con cáncer en tratamiento: ajuste, crianza parental y calidad de vida. (Tesis doctoral). Universidad de Sevilla, Sevilla. 